\title{
N89-10068
}

\section{PLAN-IT: SCHEDULING ASSISTANT FOR SOLAR SYSTEM EXPLORATION}

\author{
William C. Dias \\ ITT Federal Electric Corp. \\ Julia A. Henricks \\ Jet Propulsion Laboratory \\ California Institute of Technology \\ Jennifer C. Wong \\ Jet Propulsion Laboratory \\ California Institute of Technology
}

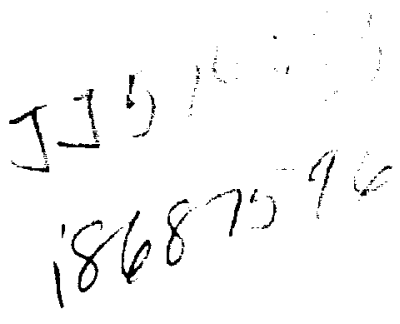

ABSTRACT

At the Jet Propulsion Laboratory (JPL), the scheduling of spacecraft activities is a complex endeavor for which streamlining is always being sought. Using the Comet Rendezvous Asteroid Flyby mission (CRAF - proposed for 1993 launch) as a development base, PLAN-IT (a framebased expert scheduling system shell) has been adapted to assist in one phase of schedule generation. PLAN-IT CRAF automatic scheduling routines attempt to return a 'good-cut' schedule which can be adjusted by an expert with time-saving graphic manipulation tools. Work to date has led to a "GO" decision on technical factors for online capability development.

\section{INTRODUCTION}

The scheduling of spacecraft activities at the Jet Propulsion Laboratory (JPL) is a very involved process requiring many personnel over long time periods $(1,2)$. The use of specialized automation tools has evolved and been encouraged over the years to increase the effectiveness and responsiveness to changes in the scheduling processes (3). Although the use of automation in the scheduling process reduces the workload of scheduling personnel, a main benefit is to gather more science data by allowing the scheduling of as many activities as possible while controlling mission risk.

Recent interest has increasingly focused on the potential of artiflcial intelligence (AI) to achieve those ends (4). Future spacecraft schedules will have a greater need for advanced scheduling automation tools because of enhanced spacecraft capabilities and the desire to automate tasks which are now labor intensive.

The scheduling of spacecraft activities may be divided into five phases (5):

1) Request Generation

2) Request Integration

3) Activity Detail Design
Collection and preprocessing of requirements

Integration of requests into a timeline

Refinement of detail in schedule 


\section{4) Sequence Generation \\ 5) Command Generation}

Translation of plan to a sequence of commands

Translation of commands to program load

A scheduling program called PLAN-IT, short for Plan-Integrated Timelines, provides general interactive scheduling capabilities using AI techniques. It has proved to be applicable to spacecraft scheduling in the Request Integration Phase. The test case for the study of this applicablitity was the Comet Rendezvous Asteroid Flyby (CRAF) mission and is the subject of this paper.

BASIC, STRUCTURED, AND EXPERT SCHEDULING TECHNIQUES

Recent literature has pointed out advantages to "evolutionary delivery" or other staged delivery of complex, innovative software systems $(6,7)$. This paper takes the view that construction of an Expert System for scheduling benefits by this treatment. A scheme for evolution of expert functionality is outlined in this section.

The art of scheduling encompasses many techniques and methods for generating a "good" schedule. Schedulers' techniques fall into three categories: Basic, Structured and Expert. One approach to development of an Expert System for a given scheduling application is to evolve from a simple to a more complex level of support. This can be done by increasing the level of intelligence being represented in the system, progressing from the Basic through the structured and finally the Expert categories of support as outlined below. Attempts to develop Expert Systems from manual systems or those automated at only a Basic support level may incur excessive and unnecessary development risk. An Expert System should encompasses not only the Expert techniques, but also the Basic and Structured techniques as described below.

Basic

The Basic category of scheduling techniques emphasizes manual control of basic operations performed on individual activities. Basic techniques focus on a single activity and not on how this activity affects other activities. Rnowledge of how a decision affects the schedule may be represented in the system and displayed graphically. That knowledge does not control execution of a command at the basic level, but may be used to report information to the operator. Using this knowledge, the operator controls execution. One of the effects of this is allowing systems to be partially functional in real environments with less completeness in the system internal knowledge.

The Basic category encompasses three functional operations: move an activity to another time frame, delete an activity, and add new activities to the schedule.

This category emphasizes manual execution which gives the user the flexibility to move activities at his discretion. The user scans the schedule and manually moves an activity to an area of lesser conflict. The strength of these techniques is best illustrated where it is desired to alter a schedule which has already been generated. 
These Basic techniques are widely used in scheduling. Systems which address this category of techniques can be applied in a wide variety of scheduling situations. An expert scheduling system 'shell' should include these basic operations.

\section{Structured}

The Structured category incorporates more knowledge of the attributes of an activity. System internal knowledge controls execution of functions to a greater degree than with the Basic category. Examples of knowledge attributes include separation criteria or precedence relations among activities. The structured category also operates coherently on groups of activities or combines the effects of several basic techniques.

The following examples of structured operations show why more knowledge about activities must be present in the system for correct execution:

1. Move activity A after activity B.

2. Adjust separation times between activities of type $C$ to a maximum of 20 minutes and a minimum of 5 minutes.

3. Insert activity $D$ between activities $E$ and $F$, and shorten its duration accordingly.

4. Repeat activity $G$ at the same time, every day of the week.

5. Reschedule activity $\mathrm{H}$ at time $\mathrm{Z}$, and automatically reschedule activities logically dependent on activity $\mathrm{H}$ accordingly.

It can be seen that operations in the structured category are more specific than those in the Basic category. There may be a subset of operations which are applicable to broad generic classes of scheduling. For instance, Operation 5 could be included in a generic scheduling tool devoted to handling problems with inter-activity temporal dependencies such as on a Pert chart. The operations in the structured category are usually very specific for a particular application.

\section{Expert}

The Expert category is characterized by operations which:

a) Encompass many activities

b) Include heuristics or 'rules of thumb' for simplifying scheduling problems

c) Generate "good" schedules by complex rules, such as:

1. Rearranging the schedule to minimize total conflict.

2. Rearranging the schedule to minimize the variance in work loads over time.

3. Delete activities, as necessary until conflicts are eliminated.

There is no hard and fast division between structured category techniques and Expert category techniques. Evolution from the Structured category to the Expert category is a low-risk means of 
evolving towards an Expert system for a specific scheduling application.

PLAN-IT OVERVIEW

This section provides an overview description of PLAN-IT, the tool used to develop the scheduler for the CRAF test case. The term "developers" used in this context refers to the people customizing PLAN-IT for a particular application. The term "user" refers to the person who will use the customized version of PLAN-IT to generate schedules.

PLAN-IT is an interactive scheduling tool. PLAN-IT is written in Zetalisp on a symbolics 3640 using version 6.1 of the operating system. PLAN-IT utilizes the frame features and object oriented programming capability provided by the zetalisp FLAVOR system. The symbolics has a high-resolution graphic screen and a three key mouse which is utilized by PLAN-IT to enhance the user interface.

The PLAN-IT screen (see Figure 1) graphically displays the activities which are scheduled and the resources the activities utilize. The PLAN-IT screen graphically represents the methods experts use to lay out a schedule. The upper portion of the PLAN-IT screen displays activities as a series of horizontal lines. Resources are represented as horizontal rectangles (thick rectangular bars) directly below the activity area. A white area in the resource line indicates that a resource is not allocated; gray implies utilization within the bounds of a given maximum; black indicates an interval of oversubscription or conflict.

Activities are internally represented with frames $(8,9)$. Resources are internally represented as blackboards $(8,10)$. Each resource line maintains internal lists of conflict and usage ratings for each interval of time. These interval bounds are determined as the point where usage differs. Any change (permanent or provisional) to an activity forces maintenance of the associated blackboards with an optional screen update.

PLAN-IT 'strategies' are blocks of code which, when invoked, assist the user in resolving or analyzing conflicts. These strategies are initiated from a pop-up menu.

Developers customizing PLAN-IT to a given application must have knowledge of PLAN-IT's internal functions and a background in zetalisp. Software to interpret the input file and to display the desired output to the end user must be generated for each unique application. Each resource timeline is defined by utilizing a series of macros developed speciflcally by the original PLAN-IT designers. Code which defines and displays conflict on the resource blackboards must be written, again with help of PLAN-IT macros. The next step is the design and implementation of software strategies to assist the user in generating a "good" schedule. Since PLAN-IT is an ongoing development, changes to the core code (i.e., below the level of the macros), may be required in a specific application when conditions are not fully representable in the macros. 

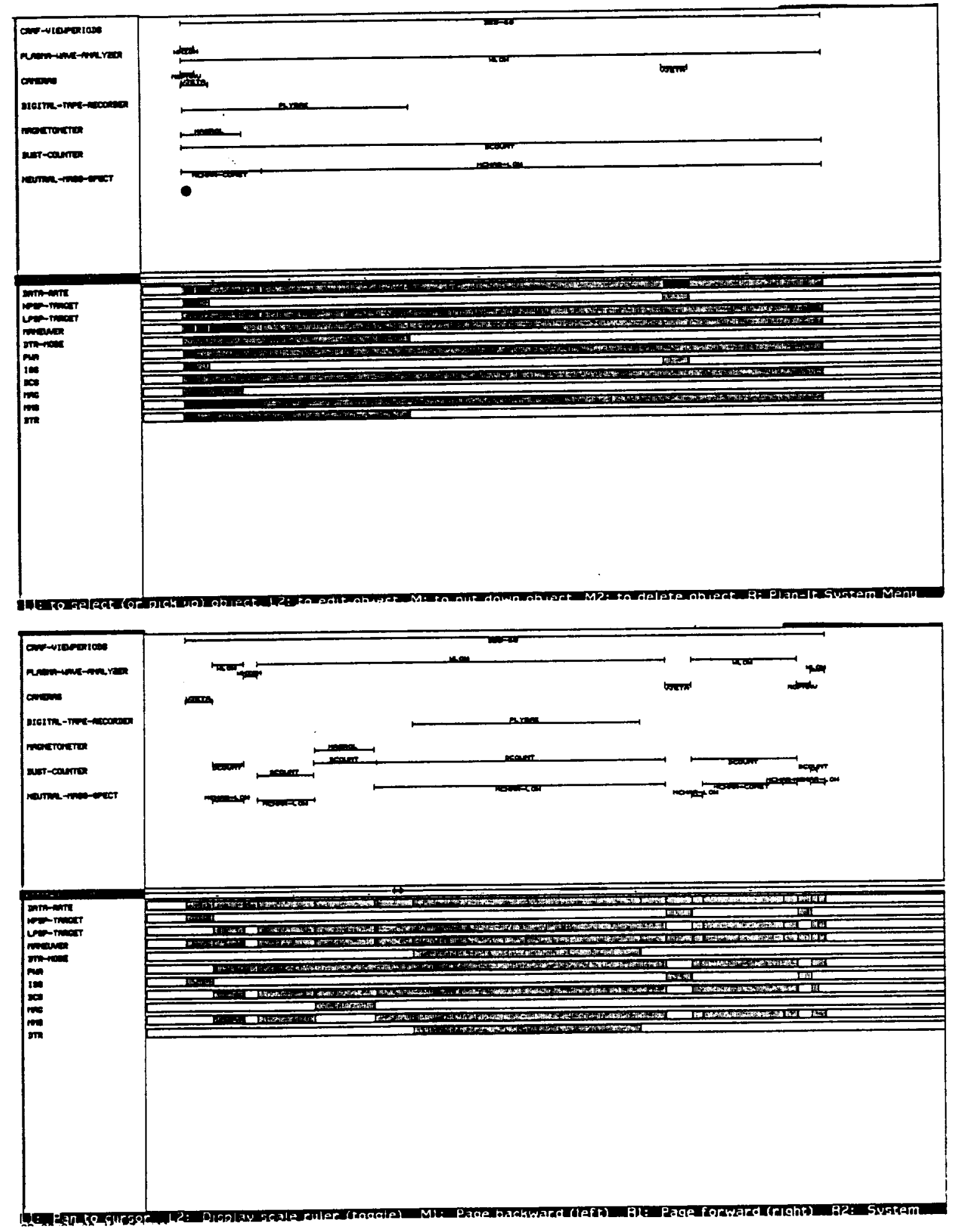

FIGURE 1 PLAN-IT SCREEN Conflicts are indicated by the solid-black bars. Top screen shows a single track as initially loaded by PLAN-IT. Bottom screen shows the same track after the conflicts have been removed. 


\section{CRAF PROBLEM OVERVIEW}

The CRAF mission has a proposed launch date of 1993 (11). After a cruise of several years with several gravitational assists from close flyby of planetary bodies and an asteroid encounter, CRAF will encounter the comet Tempel 2 near the orbit of Jupiter in 1996. After conducting preliminary science activities, CRAF will accompany the comet past perihelion (closest approach to the sun) in 1999. The early encounter phase is expected to have power resource constraints due to the combined effects of spacecraft dependency on solar power while distant from the sun, battery storage, and a small nuclear power source.

The spacecraft instruments are housed on two separately movable scan platforms, on a stationary boom, and on the spacecraft body. The instruments on platforms are fixed to those platforms, thus it is the platform that is slewed to achieve the proper orientation for a requested observation, not the instrument itself. These instruments collect science, engineering and navigational data. This data is collected at specific data rates which must either be recorded on tape or communicated to the ground via antennas in the Deep Space Network (DSN) .

Our test set consisted of nine hypothetical requests for science, navigation and engineering activities over a one week period near Tempel 2 perihelion. Each request represented multiple occurences of an activity, so the nine requests expanded to 77 activities. The activities had requirements and constraints on instrument usage, the platform pointing position, spacecraft orientation, data rate, and separation time. One request required use of the digital tape recorder (DTR) for record and playback. Ground rules for this demonstration stipulated that all activities were to be performed during the nine hypothetical CRAF DSN tracking viewperiods (See Figure 2). The data rate constraints in those tracks place limitations on the DSN link capabilities and tape playback speeds.

From the nine requests, the developers extracted the details implied by the requests and derived the definitions for conflict and constraint violation conditions. The following is a description of the extracted details.

First, of the nine requests, two were multi-phase. These two multiphase requests were expanded into five single-plase requests for a total of twelve requests. A description of the twelve requests and their attributes are found in Figure 3.

Second, requests fell into two categories: short-and long-duration. The short-duration activities were performed one or more times per week. These short-duration activities had a higher priority than activities which were requested continuously throughout the one-week period.

In contrast, the long-duration activities were requested to be continuous. Portions of these activities could be suspended in favor of the short-duration activities. 


\begin{tabular}{|l|c|r|c|c|}
\hline $\begin{array}{l}\text { DEEP } \\
\text { STACE } \\
\text { STATION }\end{array}$ & $\begin{array}{c}\text { ANTENNA } \\
\text { DIAMETER } \\
\text { (METERS) }\end{array}$ & $\begin{array}{c}\text { DATA } \\
\text { RATE } \\
\text { (KBPS) }\end{array}$ & $\begin{array}{l}\text { NUMBER OF } \\
\text { TRACKS } \\
\text { WEEK }\end{array}$ & $\begin{array}{l}\text { DURATION OF } \\
\text { EACH TRACK } \\
\text { (HOURS) }\end{array}$ \\
\hline & & & & \\
14 & 70 & 115.2 & 1 & 8 \\
15 & 34 & 40.7 & 3 & 8 \\
43 & 70 & 115.2 & 1 & 8 \\
45 & 34 & 40.7 & 2 & 8 \\
63 & 70 & 115.2 & 1 & 8 \\
65 & 34 & 40.7 & 1 & 8 \\
\hline
\end{tabular}

FIGURE 2 DEEP SPACE NETWORK TRACKING

ASSIGNMENTS FOR CRAF TEST BED 


\begin{tabular}{|c|c|c|c|c|c|c|c|}
\hline ACT IVITY & INSTRUMENT T & PLATFORM & MANEUVER & TARGET & $\begin{array}{c}\text { PATA-RATE } \\
\text { (BPS) }\end{array}$ & $\begin{array}{l}\text { PURAT ION } \\
\text { (MIN) }\end{array}$ & $\begin{array}{l}\text { PEREORMAHCE } \\
\text { CONSTRAINTS }\end{array}$ \\
\hline VTARCAL-RT & Iss & HPSP & STAT & STAR-FIELD & $115.2 \mathrm{~K}$ & 60 & once \\
\hline VTARCAL-RCD & Iss & HPSP & MNVR & CAL-P LATE & $115.2 \mathrm{~K}$ & 120 & once \\
\hline VTARCAL-PB & -- & -- & STAT & -- & $100.8 \mathrm{~K}$ & 170 & after VTARCAL \\
\hline MAGROL & MAG & $\cdots$ & MNVR & -- & 400 & 45 & once \\
\hline NOPNAV & ISS & AP SP & STAT & STAR/COMET & $115.2 \mathrm{~K}$ & 10 & once/track \\
\hline DCOUNT & $D C$ & LP SP & STAT & COMET & 44 & cont & (1) \\
\hline VJETA & ISS & HPSP & STAT & COMET-JET & $115.2 \mathrm{~K}$ & 20 & twlce/track (2) \\
\hline MCHAR-C & NMS & LP SP & STAT & COMET & 1000 & 60 & once/track \\
\hline MCHAR-A & NMS & LPSP & STAT & ANT I-COMET & 1000 & 60 & once \\
\hline MCHAR-L & MMS & LP SP & STAT & COMET & 300 & cont & --- \\
\hline WLOW & PWA & -- & $D-C$ & --- & 200 & cont & -- \\
\hline WHIGH & BWA & $\cdots$ & $D-C$ & --- & $115.2 \mathrm{~K}$ & 10 & once/track \\
\hline
\end{tabular}

DEFINITIONS :

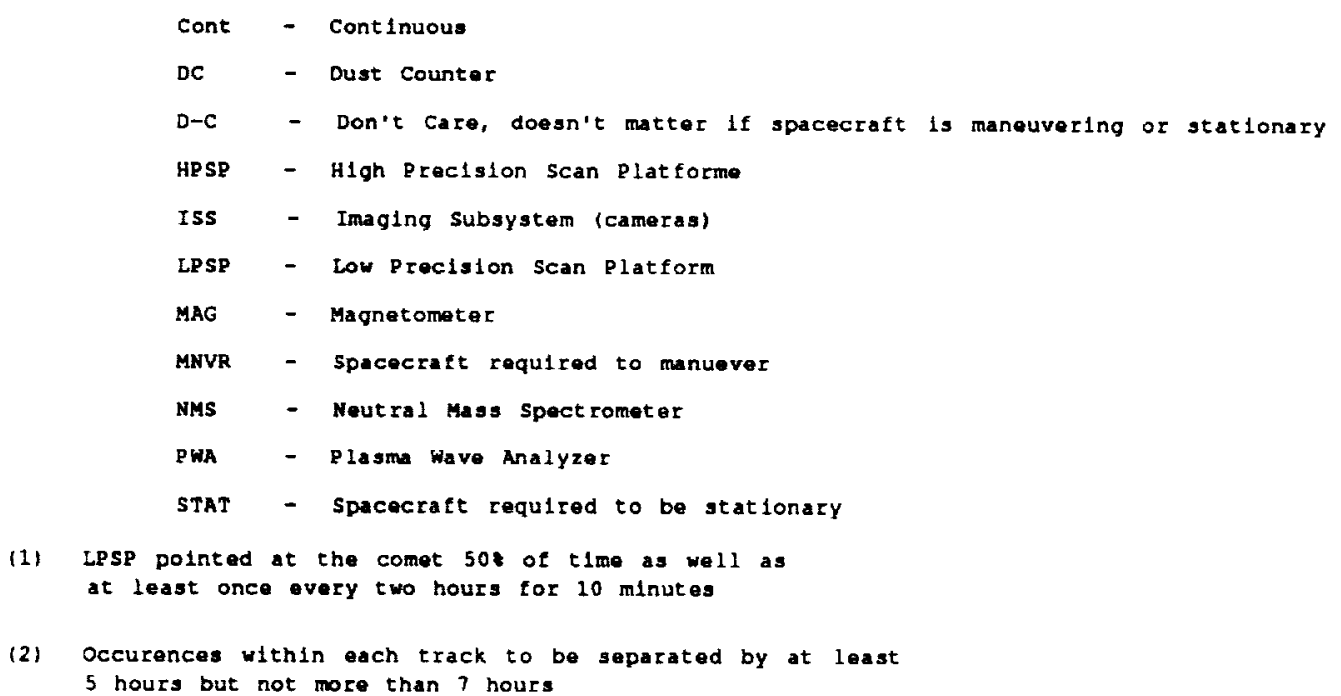


Third, from the twelve requests, definitions for conflict and constraint violation conditions were derived. Conflicts occurred when activities were scheduled in a time frame which required more resources than were available.

An example of an instrument usage conflict is two activities requiring the same instrument to be scheduled at the same time. An instrument can only accomodate one activity at a time.

An example of a constraint violation can occur with respect to spacecraft movement. An activity requiring the spacecraft to be stationary during 1ts observation cannot be scheduled in the same time frame as an activity requiring the spacecraft to be maneuvered. A more detailed description of conflict and constraint definitions is included in the next section.

\section{PLAN-IT CUSTOMIZATION FOR CRAF}

This section discusses the CRAF test case with respect to PLAN-IT. The representations of the various data elements such as activities, resources, conflicts and constraints are discussed below. The different scheduling technique categories (Basic, Structured, Expert) added to the PLAN-IT repertoire, and the rationale for selecting these techniques are reviewed.

Personnel Background

Three engineers developed the PLAN-IT overlay code for the CRAF test case over a time span of seven months. The seven month implementation period included: 1) gaining experience in LISP programming, 2) familiarization with the PLAN-IT core code, 3) defining the CRAFspecific representation and processing requirements, and 4) coding of those requirements. The engineers combined work experience included spacecraft scheduling and programming for resource allocation problems. The original PLAN-IT designers were available for consultation throughout the seven months, as were CRAF spacecraft engineers. Development efforts culminated in a PLAN-IT CRAF proof-ofconcept demonstration in October 1986, followed by informal CRAF project recognition of the product's overall potential.

\section{Activity Representation}

Activities were represented as frames with slots for each specific attribute of the activity. The attributes were a unique identifier, the start/stop times, the duration of the activity, maneuver status, platform targetting, data rate, and time windows. PLAN-IT created the activity frames as it parsed the input request data file.

Resource Conflict Representation

Resources were internally represented as blackboards. Each resource timeline contained time intervals, conflict and usage ratings. As activities were moved within the schedule, the resource blackboards updated themselves accordingly. Following is a description of each resource utilized by the CRAF activities and a description of the 
conflict and constraint conditions that were applicable to each resource.

Instrument usage was represented on the INSMRONamr OSAGE Iines (PWA, ISS, DCS, MAG, NMS resource lines in Figure 1). A unique resource line was assigned to each of the five instruments. The conflict representation associated with each of the instrument resource timelines was correspondingly simple - a single instrument usage could be requested by only one activity within any time frame. Graphically the resource timelines displayed white when the instrument is not used, gray when the instrument was requested for usage by one activity, and black when several activities requested the same instrument in the same time frame. Dedicated instrument use was assumed to be required for all CRAF activities, although in practice, scientists occasionally use the same data for multiple experiments.

The data rate resource was represented on the DarA RATE Iine. Each DSN antenna is characterized with the ability to support a spacecraft track within its maximum data rate capacity which is determined by equipment configuration and distance to the spacecraft. Figure 2 shows the data rate capacity for each test DSN track. All activities were scheduled within the given data rate envelopes. The DSN antennas can support several activities in the same time interval provided the sum of the activities' data rates does not exceed the maximum DSN Iink capacity. The DATA RATE resource line showed white when no data activity took place, gray when some bandwidth was used, and black when the maximum data rate was exceeded.

Targetted usage of instruments on the Low Precision Scan Platform (LPSP) was represented on the IPSP TARGrT line. These instruments had very general targetting criteria. General targets were symbolized by the target name, for example, EARTH, STAR and COMET. Activities were in conflict when they disagreed on general pointing requirements.

The IPSP TARGTP resource line showed gray when an instrument on the low precision scan platform had targetting requirements. Thus, the resource line showed white when none of the LPSP instruments was used or if an LPSP instrument was in use but untargetted. Black showed when conflicting targets were requested for the same time.

Usage of instruments on the High Precision Scan Platform (HPSP) was represented on the BPSP TARGET line. In contrast to the LPSP, the HPSP instruments ( $1 . e$. , the cameras) had such exact targetting criteria that no two independently requested activities could conjointly utilize the same platform orientation. Representing exact targetting criteria (azimuth and elevation angles) would require a significant coding effort beyond the coding assistance provided by the PLAN-IT macros.

For the above reasons, the HPSP TARGET line showed a conflict whenever the high precision scan platform supported two different activities concurrently, regardless of how they were targetted. In this data set, the result was somewhat trivialized by the fact that only one instrument was present on the HPSP, unlike the actual plan for CRAF which has a complement of scientific and engineering instruments on the HPSP. 
Maneuver status was represented on the MANEUVER resource line. Activities had one of the following maneuver states: YES maneuver the spacecraft, NO don't maneuver the spacecraft or DON'T CARE whether the spacecraft is manuevered or stationary. The assumptions made were:

(a) No two activities which required the spacecraft to maneuver could share the same maneuver

(b) No activity which required the spacecraft to be stationary could coexist with an activity which maneuvered, though several stationary activities could co-exist with each other

(c) Activities which don't care about maneuvering could coexist with any other activity.

Maneuver conflict code was written which stated that if two activities of the type (a) or (b) occurred, a conflict was displayed on the MANEUVER resource line. Portions of the maneuver resource ine showed gray if one or more activities requiring a stationary spacecraft were concurrent, or if a single activity requiring a maneuver was scheduled. Any activity with LPSP or HPSP targetting requirements required, by default, a stationary spacecraft unless a maneuver was specifically requested (e.g., VTARCAL).

The assumptions detalled above were in accord with the test case. Realistically, the following qualifiers should be noted:

(a) Maneuvers, such as spacecraft roll maneuvers, could sometimes be shared by several activities.

(b) The degree of "hardness" of the stationary spacecraft requirement varies.

The DTR-MODE resource line was coded to show white when no activity was taking place on the tape recorder, light gray when recording was scheduled, dark gray when data was played back, and black when recording and playback were scheduled in conflict.

\section{Besource Constraint Bepresentation}

The other major constraints included minimum and maximum separation times between activities, percentage of time pointed at specific targets, and separation time between targetted intervals of a given activity. These constraints were not represented on the resource timelines, so conflict was handled by coding special functions to run from a menu as discussed below. This meant that the code to detect these conditions could not be integrated with other interactive processing or other strategies through the PLAN-IT blackboard system.

Separation time constraints were handled by the PLAN-IT input preprocessor. It set up time windows during which activities could take place. Thus if activity-A was required to precede activity-B by 5 to 7 hours, and activity-A was placed by the preprocessor at 10:00 AM, it placed activity-B at 3:00 PM with an associated two-hour window ending at 5:00 $\mathrm{PM}$. In this 'stationary' time window, if something moved activity-A, there was no mechanism to move the window for activity-B; that would be a 'dynamic' time window, which PLAN-IT could not support except at the expense of considerable CPU time. Although code for it could be written, iterative strategies would run much 
longer. The following is a description of three constraints of this type.

Target Time Percentage Constraints were requirements to track a certain target with a certain instrument for a definite minimum percentage of the time. The underlying, unstated requirement was that the requester desired full time tracking, knowing it could not be achieved, but wanted to register the fact that when the activity was suspended, the need to resume that tracking increased with time.

Total Time Percentage Constraints were similar to Target Time Percentage Constraints, except they were untargetted. There was some overlap in coding these constraints.

Separation Time Constraints took the form of repetition requirements. Repetitive activities were required to be separated by either a minimum or a maximum amount of time or both. Again there was an overlap in coding with the constraints above.

\section{CRAF Scheduling Categories}

PLAN-IT did not contain the techniques described in the Basic, Structured and Expert categories. PLAN-IT did provide the structures for gathering the data required for implementation of the different scheduling categories. The three scheduling categories are described with respect to the PLAN-IT CRAF test case. PLAN-IT provided assistance in the development of the Basic Category techniques by providing a method by which the Basic techniques could be coded in LISP and interfaced with the existing core of code.

The Bastc techniques developed for CRAF have not yet been incorporated into generic PLAN-IT. The program's good graphic and mouse interface, when combined with application code, enhances user friendliness.

The PLAN-IT CRAF test case emphasized features which gave the user full control while generating a schedule. The Basic category operations designed and coded for PLAN-IT CRAF included:

E1 - Move moused activity to moused time.

E2 - Delete moused activity.

E3 - Examine moused activity detail.

PLAN-IT facilitated development of the structurad category techniques in the same manner as it did for the Basic category techniques. Broad classes of scheduling problems (such as a Pert chart application) have not been implemented in generic PLAN-IT. So, the developers wrote the software to accomodate the Structured techniques. The following is a description of some of the operations implemented for PLAN-IT CRAF in the Structured category.

DELETE CONFLICTING PART (AI) was used to delete any part of the moused on activity which is in conflict in any way. The strategy was to move short-duration activities around manually using El (to get them out of conflict with each other) then run A1 on each long-duration activity. This quickly creates a conflict free schedule for some tracks. 
Some activities were in conflict solely because of target and maneuver conflicts. Al could delete those activity portions, but there were cases where it would be better to relax the targetting constraint and preserve the activity itself in untargetted mode during an otherwise conflicting maneuver. For example it was better for the dust counter to be counting dust in a direction away from the comet than not counting at all. This scenario was accomplished by applying DECIMATE ACTIVITY (A2) to long-duration activities, which cut them up into ten separate descendant activities, followed by UNTARGET (A3) on the conflicting tenths, followed by RECONNECT CONTIGUOUS ACTIVITIES (A4) which would reconnect the 'decimated' activity. All these are directed by the mouse.

Another scenario was to DECIMATE all the long events, delete some of the tenths using E2, move things around manually using E1, then EXPAND EVENTS WITHOUT CONFLICT (A5) which would expand the decimated events after this manual 'shuffling' to the extent possible without instituting conflicts.

In the Expert Category different customizations of PLAN-IT have included complex 'strategies' which were attempts to code as much expertise as possible into the software. We tailored one of these, SHUFFIE TO REDUCE CONFLICT (S1), and found that it worked well to reduce conflict to an initial minimum. This minimum was a starting point for the rest of the scheduling operations.

The PLAN-IT initial allocator piles activities on top of each other at the beginning of their windows, regardless of conflict. Since many activities have windows a week long, this created an initial allocation with a lot of conflict at the beginning of the week. One heuristic we coded and tried with some success was DISTRIBUTE LOWDENSITY EVENTS (S2). It collected events which occurred less than once per track and allocated them evenly among the tracks. Its only constraint was not to put activities in tracks which could not support the requested data rate.

The combination which seemed to give the best result was to execute S2 then S1 to return a "good cut" schedule.

specialized constraint checking could not be practically represented in the blackboards, because their integration into the basic conflict definitions resulted in excessive execution time. The decision was made to have separate reporting code which was invoked by an operator call from the menu. The disadvantage was that operator decisions could cause increased conflict in these areas without the operator being the least aware of it till an explicit check was made later.

The TIME-CHECKER (C1) routine compared required separation time to actual separation time constraints for a supplied activity name, and displayed activities and time periods which violated requirements for minimum or maximum separation times.

POPUP-STATS (C2) showed a percentage of the time spent supporting an activity along with targetting percentages. 


\section{ANALYSIS OF THE CRAF DEMONSTRATION RESULTS}

The CRAF demonstration was successful. Conflicts could be eliminated and constraints were reported within a reasonable time span, and in a user-natural way. The core of the interactive scheduling capability shows sufficient promise to be worth incorporating into an operational tool, and basis exists for further development towards an Expert System for the spacecraft scheduling problem.

It was our feeling that use of the mouse and menu techniques, as opposed to keyboard input, was necessary for the successful creation of a user-natural impression. Every time the scheduler takes his eyes off the screen to input keystrokes, he loses concentration on the problem. PLAN-IT made it possible to design an interface profitably emphasizing the mouse and menu.

It is unclear whether the final CRAF science instrumentation set will present problems of a qualitatively different nature from the test set. The general observation is that each instrument tends to present a qualitatively unique scheduling challenge.

The number and complexity of constraints imposed on the range of acceptable results would likely be far greater in a real situation. This is partly a function of the number of instruments and wider variety of requests, and partly of operational experience. A wide variety of conflict and constraint conditions need to be studied to characterize the tractable conditions.

\section{FUTURE WORK}

Although the PLAN-IT CRAF initial development met its objectives, additional work is implied for the future if the ultimate objective of operational support is to be met.

There is a requirement to represent steps within activities, with temporal and precedence interrelationships among the steps. It is generally agreed the test set representation was biased in favor of single-step, single-resource activities. However this is not considered a research issue, since other PLAN-IT implementations (12) support multi-step activities.

Dr. Borls Katz (Massachusetts Institute of Technology) demonstrated a natural language parser for possible use as a front end for the PLANIT scheduling process (13). Data originating from natural language was translated to Lisp forms which were fully compatible with the CRAF test bed PLAN-IT software. Data was input, displayed and updated at high speed using the Katz parsing system. We are optimistic such capability will have a place in spacecraft scheduling pending further definition of the role for natural language in that endeavor.

The power and energy management quandary inherent in being dependent on energy collected by solar panels is likely to require a heuristic solution pecultar to the CRAF spacecraft. The resource can be modelled as a continuous function dependent on distance from the sun. 
Future work includes expansion in the Request Integration and the Activity Detail Design areas including tape recorder management. Heuristics and functions different from the test case will be required.

The current support for CRAF can be enhanced to include scheduling of activities outside tracking times.

\section{CONCLUSIONS}

The PLAN-IT CRAF effort was successful because of the efficacy of the tools afforded by PIAN-IT as a development base. PLAN-IT CRAF showed promise in its ablitity to assist interactively with integrating requests in a complex mission, using a subset of the mission resources over a subset of the conflict and constraint conditions.

With Project approval, capabilities to be added to the CRAF scheduling paradigm include power, energy and tape recorder management. Capabilities which require additional coding include activities outside of tracking times, and providing multi-step activity definitions.

Suggested improvements for the generic PLAN-IT core rose out of the CRAF effort: (1) provide Basic category commands, and (2) provide a language in which a higher-level 'applications' coder can design Structured and Expert Levels operations for his application.

\section{ACKNOWLEDGEMENTS}

The PLAN-IT CRAF demonstration described in this paper was performed by the Jet Propulsion Laboratory, California Institute of Technology, under contract with the National Aeronautics and Space Administration (NASA), and was funded through the Director's Discretionary Fund.

The PLAN-IT development was jointly funded by the NASA Office of Aeronautics and Space Technology (Code R) and the NASA Office of Space Science and Applications (Code E).

The authors wish to acknowledge the special assistance of CRAF Project representatives Sylvia Miller and Roy Kakuda for their help in comprehending the spacecraft and mission features. Thanks also to Donna Wolff, Supervisor of the Mission Planning Group, for providing and interpreting the test data.

The PLAN-IT CRAF demonstration would not have been possible without the technical assistance of Eric Biefeld, W. Curt Eggemeyer and Michael Hollander in interpreting the PLAN-IT core program.

The authors have been supported throughout the work by the efforts of the other members of the JPL Sequence Automation Research Group: Sven Grenander, Win Lombard, David Mittman, Stephen Peters, Mark Rokey, Louis Rubenstein, and John Sisino. 
(1) McLaughlin, W. I., and Wolff, D. M. "Voyager Flight Engineering: Preparing for Uranus". (AIAA-85-0287) AIAA 23rd Aerospace Sciences Meeting, January 1985.

(2) Ilnick, T. D. "Spacecraft Commanding for Unmanned Planetary M1ssions: The Uplink Process". Journal of the British Interplanetary Society, Vol 38, pp. 450-457, 1985.

(3) Planetary Expleration through the Year 2000 - A core Program. Solar System Exploration Committee of the NASA Advisory Council. 1983.

(4) Grenander, S. U. "Toward the Fully Capable AI Space Mission Planner". Aerospace America, Aug 1985.

(5) Page, D. Space Flight operations Center Sequencing Subsystem Level 4 Functional Requirements. (SSEQ0004-00-02). October 15, 1986.

(6) Gilb, T. "Evolutionary Delivery versus the 'Waterfall Model'". ACM SIGSOET Software Engineering Notes, Vol 10, No 3, p. 49.

(7) Peterson, B. "The Spiral Model of Software Development". Data-IInk, December 1985.

(8) Biefeld, E. "PLAN-IT: Knowledge Based Mission Sequencing". Proceedings of SPIE on Space Station Automation, Oct 1986, pp. $126-130$.

(9) Barr, A. and Feigenbaum, E. A. The Handbook of Artificial Intelligence. Vol I. p. 156.

(10) Nii, H. P. "Blackboard Systems: the Blackboard Model of Problem Solving and the Evolution of Blackboard Architectures." The AI Magazine Summer 1986.

(11) Miller, S. L., Bender, D. F., Stetson, D. S., and Myers, M. R. "Trajectory Options for the Comet Rendezvous Asteroid Flyby Mission". (AIAA-87-0641). AIAA 25th Aerospace Sciences Meeting, January 1987.

(12) Eggemeyer, W. C., and Bowling, A. "Deep Space Network Resource Scheduling Approach and Application".

This publication.

(13) Katz, B. and Winston, P. H. "A Two-Way Natural Language Interface". Broceedings of the European Conference on Integrated Interactive computing systems. (ECICS 82). September 1982. 